\title{
The Antioxidant Effect of Lanthanum Complex in Natural Rubber
}

\author{
Qiang-Guo LI ${ }^{1, a}$, Wei ZHENG ${ }^{1, b}$, Si-Zhu WU ${ }^{1, c^{*}}$ \\ ${ }^{1}$ State Key Laboratory of Organic-Inorganic Composites, Beijing University of Chemical Technology, \\ Beijing 100029, P.R. China \\ aqg875@163.com, b978757391@qq.com, wusz@mail.buct.edu.cn \\ ${ }^{*}$ Corresponding author
}

\begin{abstract}
Keywords: Thermal-oxidative Aging, Antioxidant, Lanthanum, Natural Rubber, Model-free Kinetic Methods, Mechanism.
\end{abstract}

\begin{abstract}
Mixed antioxidants composed of antioxidant $N$-phenyl- $N$ '-isopropyl-p-phenylenediamine (IPPD) and lanthanum (La) complex were added as a filler to prepare natural rubber (NR) composites. By mechanical testing, $X$-ray photoelectron spectroscopy, and thermogravimetric analysis, a string of data, including the mechanical properties, the variation of chemical surface characterization, and the thermo-oxidative decomposition kinetics parameters, was presented in this article to study the antioxidant effect of the mixed antioxidants in NR. The density function theoretical calculations were also used as an assistant to study the thermo-oxidative aging mechanism of NR and the different function mechanisms of antioxidants IPPD and La complex. The data revealed that except for functioning as a labile-hydrogen doner which was similar to antioxidant IPPD, this La complex had strong coordination abilities and large coordination numbers, resulting in the high efficiency in enhancing the thermal-oxidative stability of NR.
\end{abstract}

\section{Introduction}

Natural rubber (NR) is widely applied in many fields due to its excellent properties. But it is easily attacked by oxygen, especially under thermal condition. The thermal-oxidative aging of NR will lead to the deterioration of its properties[1]. Thus, the thermal-oxidative stability is essential for the processing and applications of NR. So far, adding antioxidants has been found to be one of the most convenient methods to retard or prevent the thermal-oxidative aging of rubbers[2].

Recent studies have found that coordination compounds composed of rare earth metals and certain organic compounds containing $\mathrm{O}, \mathrm{S}$ and $\mathrm{N}$ atoms could exert excellent long-term protective effect on the thermal oxidation process of rubbers[3,4]. The thermal-oxidative aging of NR follows an autocatalytic, free radical chain reaction mechanism[3]. With shell structure like 4f0-145d0-106s2, lanthanide series rare earth ions, whose atom numbers are from 57 to 71 , have long atom radius and a large quantity of unoccupied orbits, resulting in strong abilities in scavenging free radicals generated in the thermal-oxidative process[5].

This study made a comparative study of thermal-oxidative stability for two NR samples - one with the antioxidant N-phenyl-N'-isopropyl-p-phenylenediamine (IPPD) and the other with the combination of antioxidant IPPD and lanthanum (La) complex. The combination of experiments and simulations clarified the variations of properties and structures, and the corresponding function mechanisms of antioxidants IPPD and La complex.

\section{Experimental}

\section{Materials}

The base formulation of the two NR composites was described as follows (phr): RSS, 70; E-SBR 1500, 30; zinc oxide, 5; stearic acid, 2; antioxidants, 3; silica-VN3, 40; Si69, 4; Carbon black 234, 5; sulfur, 1.8; accelerator CZ, 1.5. In addition, $3 \mathrm{phr}$ of antioxidant IPPD was added into the base 
formulation to prepare Sample A, while $3 \mathrm{phr}$ of mixed antioxidants (antioxidant IPPD, 2.25; p-ASALa, 0.75) was added to the base formulation to prepare Sample B.

The above ingredients were mixed on a two-roll mill (Guang-dong Zhanjiang Rubber and Plastic Machinery Factory, China). The curing characteristics were determined by a discoscillating rheometer (Beijing Huanfeng Mechanical Factory, China). Then the compounds were cured in a 25T hot compression mold (Shanghai Rubber Machinery Factory, China) at $143^{\circ} \mathrm{C}$ for t90 to gain NR composites.

\section{Experimental Methods}

NR samples were thermally aged at the center of an air-circulated cabinet oven at $80{ }^{\circ} \mathrm{C}$ and atmospheric pressure. The mechanical properties were measured according to Chinese standard GB/T 528-2009. The $X$-ray photoelectron spectroscopy (XPS) measurements were made on an Escalab 250Xi $X$-ray photoelectron spectrometer (Thermo Fisher Scientific Inc., USA) equipped with monochromatic $\mathrm{Al} \mathrm{K \alpha}$ radiation source as the excitation. The analyses of overlapping peak resolving were made by XPSPEAK41 software. The non-isothermal thermogravimetric (TG) measurements at different heating rates $(5 \mathrm{~K} / \mathrm{min}, 10 \mathrm{~K} / \mathrm{min}, 20 \mathrm{~K} / \mathrm{min}$, and $30 \mathrm{~K} / \mathrm{min}$ ) were performed on a Thermo Gravimetric Analyzer (TGA)/Differential Scanning Calorimetry (DSC) calorimeter (Mettler-Toledo Co., Switzerland). The samples were heated under an air flow of $50 \mathrm{~mL} / \mathrm{min}$.

\section{Simulation Details}

All the theoretical computation results were performed using the $\mathrm{DMol}^{3}$ module of the Materials Studio ${ }^{\circledR}$ suite of software[6]. Geometry optimizations for all the structures were simulated using Perdew-Burke-Ernzerhof function, all electron type of core treatment, and a triple numerical atomic orbital augmented by additional polarization functions basis set. The free energy for a dissociation energy at $298 \mathrm{~K}\left(\Delta G^{298 K}\right)$ was calculated as follows[3]:

$$
\Delta G^{298.15 K}=G_{A \bullet}^{298.15 K}+G_{H \bullet}^{298.15 K}-G_{A H}^{298.15 K}
$$

Where $G_{A}{ }^{298.15 K}$ and $G_{H}{ }^{298.15 K}$ are the energies of two free radicals generated by the hemolysis of the covalent bond at $298.15 \mathrm{~K}$, and $G_{A H}{ }^{298.15 K}$ is the energy of the compound $\mathrm{AH}$ at $298.15 \mathrm{~K}$.

\section{Results and Discussion}

\section{Mechanical Properties}

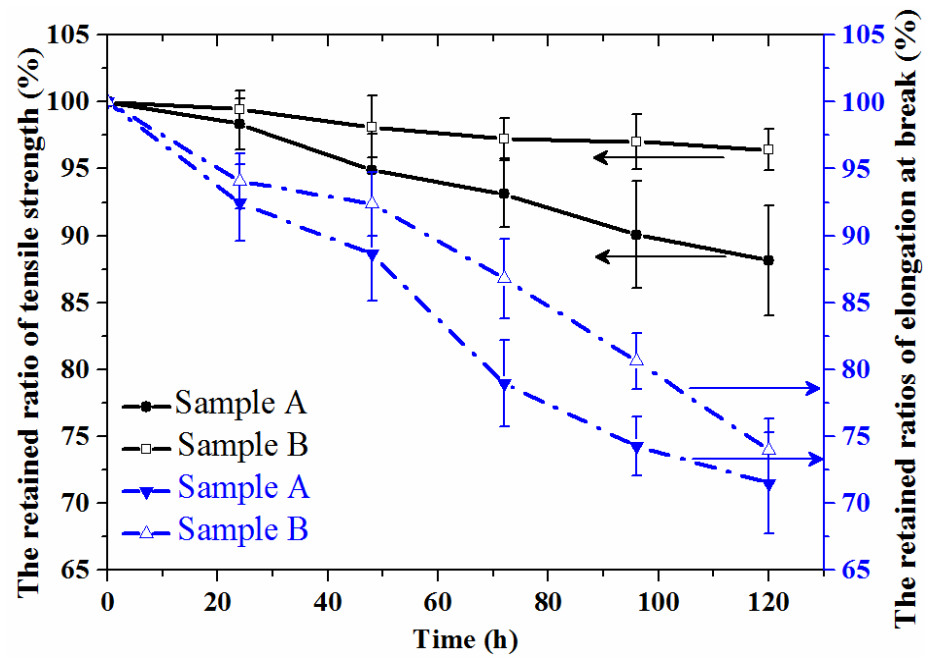

Fig. 1 The changing trends of retained ratios of tensile strength and elongation at break for the two NR samples.

Fig. 1 shows the changing trends of mechanical properties for the two NR samples. During $120 \mathrm{~h}$ 
of thermal-oxidative exposure, the retained ratios of tensile strength and elongation at break for the two NR samples both decrease as time goes by. Besides, the rate of mechanical properties reduction for Sample A is faster than that for Sample B, indicating that the addition of p-ASALa improves the antioxidant effect for NR.

\section{Chemical Surface Characterization}

The chemical surface characterization of two NR samples are quantitatively analyzed by XPS measurements. After aging at $353 \mathrm{~K}$ for $144 \mathrm{~h}$, the mole ratio of oxygen atom to carbon atom $(\mathrm{n}(\mathrm{O}) / \mathrm{n}(\mathrm{C}))$ for Sample A changes from 0.180 to 0.231; and $(\mathrm{n}(\mathrm{O}) / \mathrm{n}(\mathrm{C}))$ for Sample B changes from 0.167 to 0.190 .The detailed XPS $\mathrm{C}_{1 \mathrm{~s}}$ core-level spectra are shown in Fig. 2. Four types of carbon atoms, including C-C and C-H $(284.8 \mathrm{eV}), \mathrm{C}-\mathrm{O}(286.1 \mathrm{eV}), \mathrm{C}=\mathrm{O}(287.8 \mathrm{eV})$, and $\mathrm{O}=\mathrm{C}-\mathrm{O}(289.1 \mathrm{eV})$, exist in two NR samples. The fractions (the area for the peak of a certain carbon atom divided by the total area) of these four types of carbon atoms are shown in Table 1 . After aging at $353 \mathrm{~K}$ for $144 \mathrm{~h}$, the fractions of oxygenic carbon atoms in Sample B are all lower than those in Sample A, indicating that p-ASALa shows good antioxidant effect in improving the thermal-oxidative stability of NR by delaying the generation of thermal-oxidative aging products, e.g., ketone, ether, and ester.
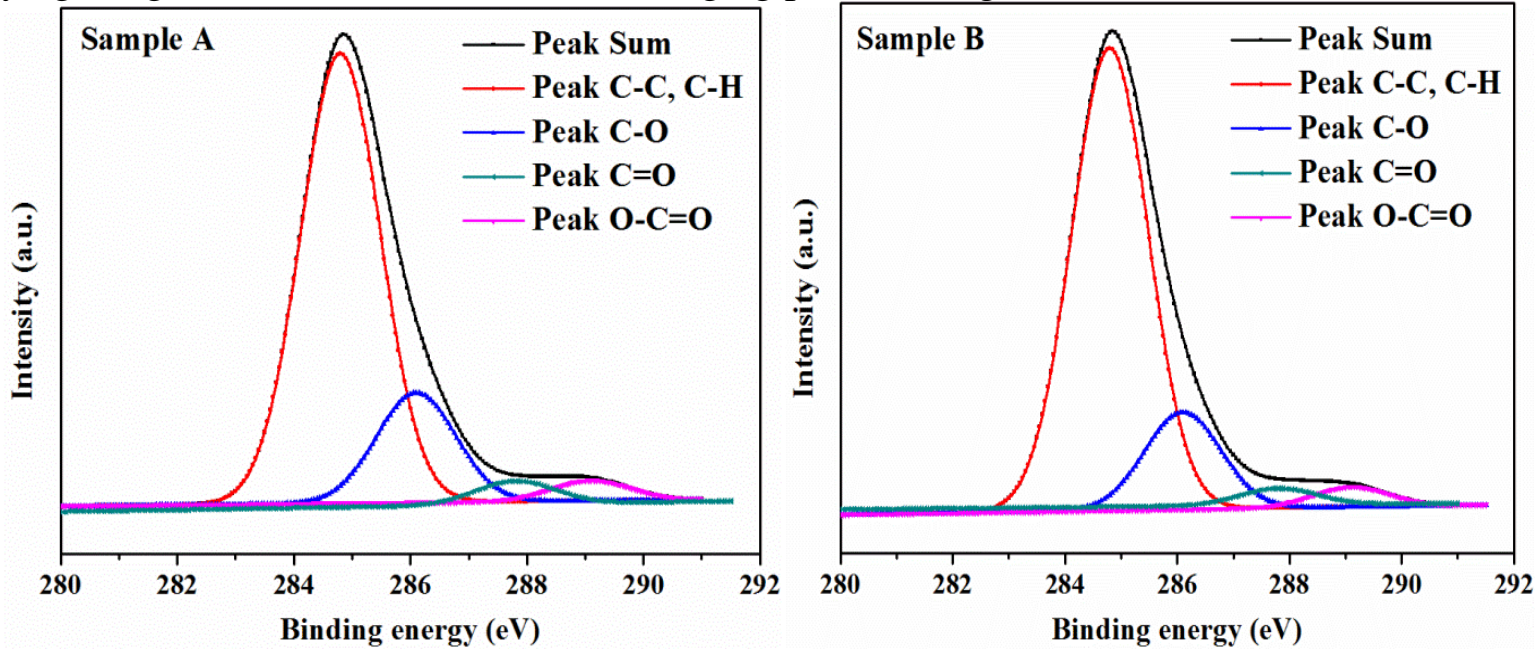

Fig. 2 XPS C1s core-level spectra of two NR samples after aging at $353 \mathrm{~K}$ for 144h: (a) Sample A; (b) Sample B.

Tab. 1 XPS analyses for the surface characterizations of two NR samples after aging at $353 \mathrm{~K}$ for $144 \mathrm{~h}$.

\begin{tabular}{c|cccc}
\hline Sample & C-C, C-H & C-O & C $=\mathrm{O}$ & O-C $=\mathrm{O}$ \\
\hline A & 0.745 & 0.182 & 0.035 & 0.038 \\
\hline B & 0.775 & 0.163 & 0.030 & 0.032 \\
\hline
\end{tabular}

\section{Kinetic Analysis}

The non-isothermal, isoconversional Flynn-Wall-Ozawa (FWO) method analyzed by TGA curves heating at different rates was proposed to determine the kinetic parameters of the thermo-oxidative degradation of NR. This method is based on the Doyle[7] approximation for heterogeneous chemical reactions:

$$
\log \beta=\log \left(\frac{A \cdot E_{a}}{R \cdot G(\alpha)}\right)-2.315-0.4567 \frac{E_{a}}{R T}
$$

Where $\beta$ is the heating rate, $A$ is a pre-exponential factor, $E_{a}$ is the activation energy, $R$ is the gas constant, $\alpha$ is the fractional mass loss, and $G(\alpha)$ is a conversional function. For a certain $\alpha, G(\alpha)$ is fixed. Therefore, $E_{a}$ can be estimated from the slope of the straight line obtained by plotting $\log \beta$ vs $T^{1}$. 


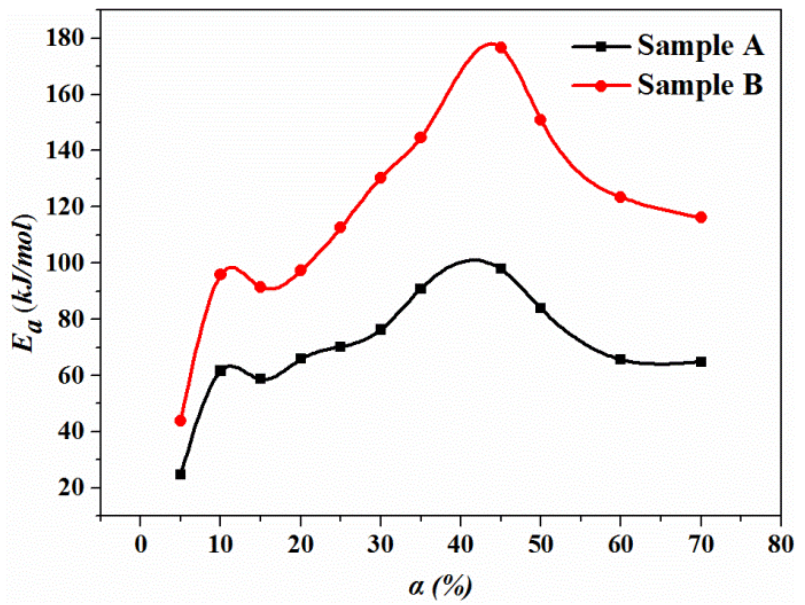

Fig. 3 Plots of $E_{a}$ versus specified $\alpha$ determined by FWO method.

Fig. 3 shows that each curve has two maximal points, dividing the thermal-oxidative degradation of NR into three stages[5]. In the first stage, $E_{a}$ increases with $\alpha$ until $\alpha$ reaches $10 \%$, corresponding to the initiation of the chain. In the second stage, when $\alpha$ is from $10 \%$ to $15 \%, E_{a}$ decreases with $\alpha$ because of the autocatalytic oxidation process during thermo-oxidative degradation. In the final stage, at $\alpha$ higher than $15 \%, E_{a}$ increases with $\alpha$ again, reaches a maximum at $\alpha$ of nearly $40 \%$, and then decreases with $\alpha$. This final stage is associated with the decomposition of thermo-oxidative products and the intensification of the thermo-oxidative degradation process. Furthermore, the values of $E_{a}$ required for thermal-oxidative degradation at every specified $\alpha$ of Sample B are higher than those of Sample A, indicating that p-ASALa has better antioxidant effect than antioxidant IPPD in NR.

\section{Different Function Mechanisms of Two Kinds of Antioxidants}

By decreasing the concentrations of the active free radicals by preferential reaction relative to NR[8], the antioxidants IPPD and p-ASALa lower the rate of NR oxidation. However, their function mechanisms and efficiencies are different.

Tab. 2 The $\Delta G^{298.15 K}$ values of C-H bonds in NR at positions (a), (b), (c) and (d); and N-H bonds in antioxidant IPPD at positions (e) and (f).

\begin{tabular}{|c|c|c|}
\hline Position & $\triangle \mathrm{G}^{298.15 \mathrm{~K}}[9]^{\exp }(\mathrm{kJ} / \mathrm{mol})$ & $\triangle \mathrm{G}^{298.15 \mathrm{~K} \operatorname{sim}}(\mathrm{kJ} / \mathrm{mol})$ \\
\hline (a) & 322.61 & 345.81 \\
\hline (b) & - & 435.97 \\
\hline (c) & 351.48 & 424.65 \\
\hline (d) & 335.58 & 354.34 \\
\hline (e) & - & 304.86 \\
\hline (f) & - & 324.22 \\
\hline
\end{tabular}
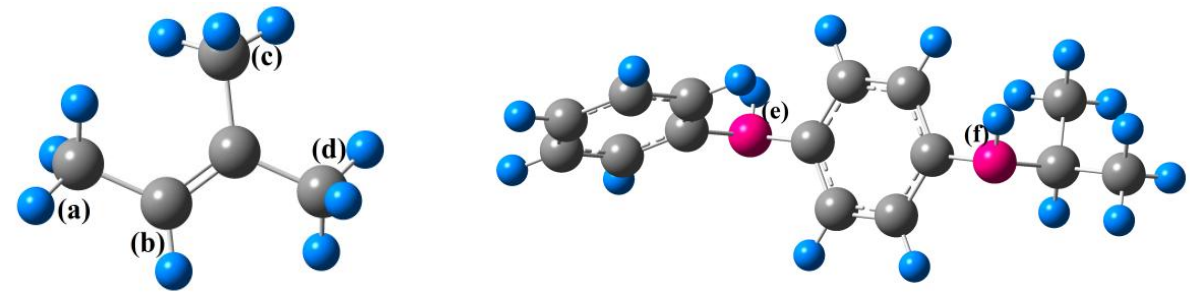

The position (a), (b) and (c) of C-H bonds and the position (d) and (e) of antioxidant IPPD are as above (blue sphere represents $\mathrm{H}$ atom, grey sphere represents $\mathrm{C}$ atom, and pink sphere represents $\mathrm{N}$ atom)

The principal mechanism of the thermo-oxidative aging of NR involves an autocatalytic, free 
radical chain reaction. The first step is the initiation of the chain. As a typical amine antioxidant, antioxidant IPPD has an active hydrogen atom $(\mathrm{H})$ in imino group(e). With the less $\Delta G^{298.15 K}$ value at position (e) than the $\Delta G^{298.15 K}$ value at position (a) of NR, antioxidant IPPD can function as a labile-hydrogen doner to compete effectively with NR chains for reactions with propagating peroxy radicals. The main reactions in which the antioxidant IPPD is involved are shown in Scheme 1.

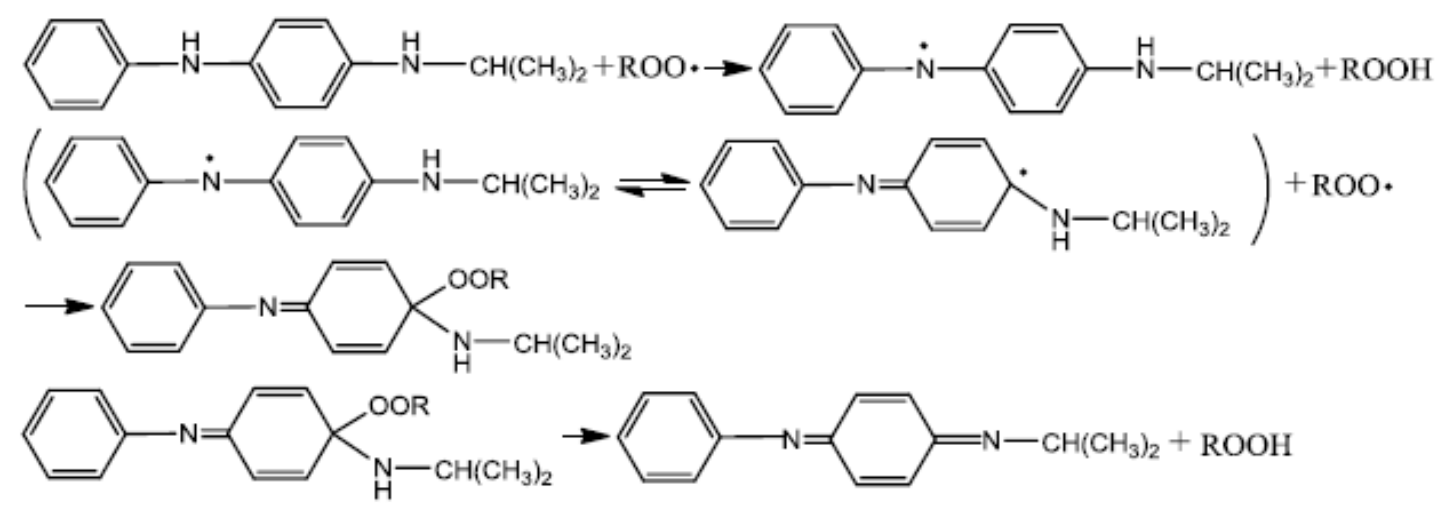

Scheme 1 The function mechanism of antioxidant IPPD (R represents alkyl).

According to Scheme 1, we can conclude that the 1 mole antioxidant IPPDs (molecular weight=226) contain 2 mole labile hydrogens, each of which can scavenge 1 mole peroxy radicals to terminate 1 mole free radical chain reactions.

The existence of two primary amine groups of p-ASALa shows the similar reason as labile-hydrogen doners in protecting NR against autoxidation. It means that 1 mole p-ASALas contain 4 mole labile hydrogens and can terminate 4 mole free radical chain reactions. Besides, with the electron shell structure of $5 \mathrm{~d} 16 \mathrm{~s} 2$, La has a full empty $4 \mathrm{f}$ orbit and a long atom radius, resulting in strong coordination abilities and large coordination numbers, which exceed 6 , normally are 7, 8, 9, and 10. Therefore, the same mass of p-ASALa (molecular weight=519) is able to scavenge more peroxy radicals and has better antioxidant effect than antioxidant IPPD to improve the thermal-oxidative stability of NR.

\section{Summary}

After thermal-oxidative exposure, NR sample B with mixed antioxidants composed of antioxidant IPPD and lanthanum (La) complex exhibited better mechanical properties and lower increases of three types of carbon-oxygen groups than Sample A with pure antioxidant IPPD. In the thermal-oxidative degradation process, the activation energy of Sample B at every specified fractional mass loss was higher than that of Sample A. The results indicated that p-ASALa had better antioxidant effect than antioxidant IPPD in NR.

The theoretical calculation results showed that 1 mole antioxidant IPPDs could doner 2 mole labile hydrogens. By effectively competing with hydrogen at position (a) in NR chain, 1 mole antioxidant IPPDs could scavenge two peroxy radicals to terminate two free radical chain reactions. Except for the two primary amine groups which could doner 4 mole labile hydrogens, p-ASALa had strong coordination abilities and large coordination numbers, resulting in the high efficiency of p-ASALa in enhancing the thermal-oxidative stability of NR.

\section{Acknowledgements}

The financial supports from the National Natural Science Foundation of China under Grant No. 51473012 and the Major Research plan from the Ministry of Science and Technology of China under Grant No. 2014BAE14B01 are gratefully acknowledged. 


\section{References}

[1] M.T. Ramesan, T.K.M. Kumar, R. Alex, B. Kuriakose, Investigations on the addition of styrene butadiene rubber in natural rubber and dichlorocarbene modified styrene butadiene rubber blends, J. Mater. Sci. 37 (2002) 109-116.

[2] M. Komethi, N. Othman, H. Ismail, S. Sasidharan, Comparative study on natural antioxidant as an aging retardant for natural rubber vulcanizates, J. Appl. Polym. Sci. 124 (2012) 1490-1500.

[3] W. Zheng, L. Liu, X. Zhao, J. He, A. Wang, T.W. Chan, S. Wu, Effects of lanthanum complex on the thermal-oxidative aging of natural rubber, Polym. Degrad. Stab. 120 (2015) 377-383.

[4] L. Fang, Y. Song, X. Zhu, Q. Zheng, Influence of lanthanum stearate as a co-stabilizer on stabilization efficiency of calcium/zinc stabilizers to polyvinyl chloride, Polym. Degrad. Stab. 94 (2009) 845-850.

[5] Xie C, Jia Z, Luo Y. Antioxidant effect of Sm(III) complex with 2-mercaptobenzimidazole in natural rubber vulcanizates. Acta Polym Sin. (2011) doi:10.3724/SP.J.1105. 2011.10090.

[6] http://accelrys.com/products/materials-studio/index.html.

[7] C.D. Doyle, Series approximations to the equation of thermogravimetric data, Nature 207 (1965) 290-291.

[8] G.Y. Li, J.L. Koenig, FTIR imaging of oxidation of polyisoprene 2. The role of N-phenyl-N'-dimethyl-butyl-p-phenylenediamine antioxidant, Polym. Degrad. Stab. 81 (2003) 377-385. 